\title{
THE TORELLI GEOMETRY AND ITS APPLICATIONS RESEARCH ANNOUNCEMENT
}

\author{
Benson Farb and Nikolai V. IVAnov
}

Let $S$ be a closed orientable surface of genus $g$. The mapping class group $\operatorname{Mod}(S)$ of $S$ is defined as the group of isotopy classes of orientation-preserving diffeomorphisms $S \rightarrow S$. We will need also the extended mapping class group, $\operatorname{Mod}^{ \pm}(S)$ of $S$, which is defined as the group of isotopy classes of all diffeomorphisms $S \rightarrow S$. Let us fix an orientation of $S$. Then the algebraic intersection number provides a nondegenerate, skew-symmetric, bilinear form on $H=H_{1}(S, \mathbf{Z})$, called the intersection form. The natural action of $\operatorname{Mod}(S)$ on $H$ preserves the intersection form. If we fix a symplectic basis in $H$, then we can identify the group of symplectic automorphisms of $H$ with the integral symplectic group $\operatorname{Sp}(2 g, \mathbf{Z})$ and the action of $\operatorname{Mod}_{S}$ on $H$ leads to a natural surjective homomorphism

$$
\operatorname{Mod}(S) \longrightarrow \operatorname{Sp}(2 g, \mathbf{Z})
$$

The Torelli group of $S$, denoted by $\mathcal{I}_{S}$, is defined as the kernel of this homomorphism; that is, $\mathcal{I}_{S}$ is the subgroup of $\operatorname{Mod}(S)$ consisting of elements which act trivially on $H_{1}(S, \mathbf{Z})$. In particular, we have the following well-known exact sequence

$$
1 \longrightarrow \mathcal{I}_{S} \longrightarrow \operatorname{Mod}(S) \longrightarrow \operatorname{Sp}(2 g, \mathbf{Z}) \longrightarrow 1
$$

The group $\mathcal{I}_{S}$ plays an important role both in algebraic geometry and in lowdimensional topology. At the same time most of the basic questions about $\mathcal{I}_{S}$ remain open. See, e.g., [Jo1] and [Hain] for surveys. For example, while $\mathcal{I}_{S}$ is finitely generated if the genus $g \geq 3$ by a theorem of D. Johnson [Jo2], it is not known whether or not $\mathcal{I}_{S}$ has a finite presentation if $g \geq 3$.

In this note we introduce a new geometric object related to the Torelli group $\mathcal{I}_{S}$, which we call the Torelli geometry $\mathcal{T} \mathcal{G}(S)$ of $S$ and announce several results about $\mathcal{T} \mathcal{G}(S)$ and $\mathcal{I}_{S}$. Our main result about $\mathcal{T} \mathcal{G}(S)$ gives a complete description of its automorphisms (they are all induced by diffeomorphisms of $S$ ). We also give a purely algebraic characterization of some geometrically defined elements of $\mathcal{I}_{S}$, namely, of the (powers of the) so-called bounding twists and bounding

Received June 15, 2004.

The first author was supported in part by NSF grants DMS-9704640 and DMS-0244542. He would also like to thank the Institut de Mathématiques de Bourgogne for its hospitality and support during the writing of this paper.

This work was done during the visit of the second author to the University of Chicago. He would like to thank the Department of Mathematics of the University of Chicago and the first author for the hospitality. 
pairs, and also of some geometrically defined collections of such elements. When combined with the description of the automorphisms of $\mathcal{T} \mathcal{G}(S)$, these characterizations lead to several algebraic results about the Torelli group $\mathcal{I}_{S}$, among which are the following.

- The automorphism group of $\mathcal{I}(S)$ is isomorphic to the extended mapping class group $\operatorname{Mod}^{ \pm}(S)$; the outer automorphism group $\operatorname{Out}(\mathcal{I}(S))$ contains the integral symplectic group $\operatorname{Sp}(2 g, \mathbf{Z})$ as a subgroup of index 2 .

- The abstract commensurator $\operatorname{Comm}(\mathcal{I}(S))$ is isomorphic to the extended mapping class group $\operatorname{Mod}^{ \pm}(S)$.

- The Torelli group is not arithmetic.

The last result is known. Actually, any normal subgroup of $\operatorname{Mod}(S)$ was proved to be non-arithmetic in [Iv3]; for more general results, proving a conjecture of the second author, see $[\mathrm{FM}]$. However, we feel that our proof sheds a new light on this result. The details of the proofs will appear in [FI].

Notes. McCarthy-Vautaw $[\mathrm{McV}]$ have extended the first corollary to genus $g \geq 3$; here with automorphisms, in contrast with commensurations, one can use relations in $\mathcal{I}(S)$. Brendle-Margalit then built on our methods to extend the much harder second result to genus $g \geq 4$; indeed they were even able to $\operatorname{compute} \operatorname{Comm}\left(\mathcal{K}_{g}\right)$ for the Johnson kernel - see $[\mathrm{BM}]$.

Torelli Geometry. A recurring theme in the theories of both finite and infinite groups is the investigation of a group by using its action on an appropriate $g e$ ometry. We will use the term geometry in a narrow sense of a simplicial complex with some additional structure, namely, with some vertices and simplices marked by some colors. For example, the main construction of Bruhat-Tits theory applied to $\mathrm{SL}_{n}\left(\mathbf{Q}_{p}\right), n>2$ gives an $(n-1)$-dimensional simplicial complex $X_{n, p}$ (with marked vertices) whose group of simplicial automorphisms preserving the marking is isomorphic to $\mathrm{SL}_{n}\left(\mathbf{Q}_{p}\right)$. Similar constructions occur in the theory of finite simple groups.

For the mapping class group $\operatorname{Mod}(S)$ of a closed surface $S$, a useful geometry is the complex of curves, denoted $\mathcal{C C}(S)$. This simplicial complex, introduced by W. Harvey [Harv], is defined in the following way: $\mathcal{C C}(S)$ has one vertex for each isotopy class of simple (i.e. embedded) closed curve on $S$; further, $k+1$ distinct vertices of $\mathcal{C C}(S)$ form a $k$-simplex if the corresponding isotopy classes can be represented by disjoint curves. It is easy to see that $\mathcal{C C}(S)$ has dimension $3 g-4$; in contrast with the classical situations it is not locally finite.

The complex $\mathcal{C C}(S)$ is a fundamental tool in the low-dimensional topology. The following theorem, which strongly supports the claim of $\mathcal{C C}(S)$ for being the right geometry for $\operatorname{Mod}(S)$, is of special importance for us.

Theorem 1 (Ivanov [Iv5]). Let $S$ be closed and let $\operatorname{genus}(S) \geq 2$. Then the group of simplicial automorphisms of $\mathcal{C C}(S)$ is isomorphic to the extended mapping class group $\operatorname{Mod}^{ \pm}(S)$. 
Our definition of a geometry for the Torelli group is guided by the definition of the complex of curves $\mathcal{C C}(S)$. In order to arrive at a complex intimately related to the Torelli group, it is natural to use as vertices the isotopy classes of separating curves and of bounding pairs, since they correspond to the natural generators of the Torelli group. By a separating curve $\gamma \subset S$ we mean a homologically trivial curve, or equivalently, a curve which bounds a subsurface in $S$. A bounding pair in $S$ is a pair of disjoint, non-isotopic, nonseparating curves in $S$ whose union bounds a subsurface in $S$. It turns out that it is useful also to introduce a marking.

Definition (Torelli geometry). The Torelli geometry of $S$, denoted $\mathcal{T} \mathcal{G}(S)$, is the following simplicial complex with additional structure:

Vertices of $\mathcal{T} \mathcal{G}(S)$ : The vertices of $\mathcal{T} \mathcal{G}(S)$ consist of:

1. Isotopy classes of separating curves in $S$, and

2. Isotopy classes of bounding pairs in $S$.

Simplices of $\mathcal{T} \mathcal{G}(S)$ : A collection of $k \geq 2$ vertices in $\mathcal{T G}(S)$ forms a $(k-1)$ simplex if these vertices have representatives which are mutually non-isotopic and disjoint.

We also endow $\mathcal{T} \mathcal{G}(S)$ with a marking, which consists of the following two pieces of data:

\section{Marking for $\mathcal{T} \mathcal{G}(S)$ :}

1. Each vertex in $\mathcal{T} \mathcal{G}(S)$ is marked by its type: separating curve or bounding pair.

2. A 2-simplex $\Delta$ in $\mathcal{T} \mathcal{G}(S)$ is marked if there are 3 non-isotopic, disjoint, nonseparating curves $\gamma_{1}, \gamma_{2}, \gamma_{3}$ so that the vertices of $\Delta$ are bounding pairs $\gamma_{i} \cup \gamma_{i+1}$ for $i=1,2,3(\bmod 3)$.

It is easy to see that the $\operatorname{group} \operatorname{Mod}(S)$ acts on $\mathcal{T} \mathcal{G}(S)$ by automorphisms, by which we mean simplicial automorphisms preserving the marking. Our main result about $\mathcal{T} \mathcal{G}(S)$ is the following.

Theorem 2 (Automorphisms of $\mathcal{T} \mathcal{G}(\boldsymbol{S})$ ). Suppose genus $(S) \geq 5$. Then every automorphism of the Torelli geometry $T(S)$ is induced by a diffeomorphism of S. Further, the natural map

$$
\operatorname{Mod}^{ \pm}(S) \longrightarrow \operatorname{Aut}(\mathcal{T} \mathcal{G}(S))
$$

is an isomorphism.

The fact that $\operatorname{Aut}(\mathcal{T} \mathcal{G}(S))$ is the extended mapping class group and not the Torelli group itself reflects intrinsic extra symmetries of the Torelli group; indeed, exactly for this reason Theorem 2 will allow us to compute the automorphisms group of $\mathcal{T} \mathcal{G}(S)$ and the abstract commensurator of $\mathcal{T} \mathcal{G}(S)$. 
Connectedness theorems. A key ingredient in the proof of Theorem 2 is the following result, which we believe is of independent interest.

Theorem $3(\mathcal{T} \mathcal{G}(\boldsymbol{S})$ is connected). For a closed orientable surface $S$ of $\operatorname{genus}(S) \geq 3$ the complex $\mathcal{T} \mathcal{G}(S)$ is connected.

There is a basic and useful subcomplex of the complex of curves, which is in fact a subcomplex of $\mathcal{T} \mathcal{G}(S)$ for closed $S$.

Definition $\left(\mathcal{C}_{\text {sep }}(\boldsymbol{R})\right)$. Let $R$ be any compact orientable surface, perhaps with non-empty boundary. We define $\mathcal{C}_{\text {sep }}(R)$ to be the simplicial complex whose vertices consist of isotopy classes of curves in $R$ which bound a subsurface of positive genus with one boundary component, and with $k+1$ distinct vertices forming a $k$-simplex if they can be represented by disjoint curves on $S$.

Note that, for a closed surface $S$, the complex $\mathcal{C}_{\text {sep }}(S)$ is just the subcomplex of $\mathcal{C C}(S)$ spanned by the (isotopy classes of) separating curves.

Theorem $4\left(\mathcal{C}_{\text {sep }}(\boldsymbol{R})\right.$ is connected $)$. Suppose that $R$ is a compact surface with $\operatorname{genus}(R)$

$\geq 3$. Then $\mathcal{C}_{\text {sep }}(R)$ is connected.

Note that if $\gamma \subset R$ is a separating curve, and genus $(R) \geq 3$, then $R \backslash \gamma$ has one component of genus at least two; in particular there is a separating curve $\beta \in R$ disjoint from $\gamma$ and bounding a subsurface of genus one. We will call such separating curves the genus one separating curves. Hence Theorem 4 follows from the following stronger result which we prove:

For genus $(R) \geq 3$, the full subcomplex of $\mathcal{C}_{\text {sep }}(R)$ spanned by the (isotopy classes of) genus one separating curves is connected.

Notice that both connectedness theorems do not hold if we omit the restriction on the genus; in fact, if the genus is equal to 2 , then both complexes have an infinite number of vertices and no edges. A new proof of this last result has been given by Masur-Schleimer [MS].

The idea of proof of Theorem 2. The main step in the proof of Theorem 2 is to show that every automorphism of the Torelli geometry $\mathcal{T} \mathcal{G}(S)$ canonically induces an automorphism of the complex of curves $\mathcal{C C}(S)$ when genus $(S) \geq 5$. Once this is proved, we can quote Ivanov's Theorem (Theorem 1 above) and complete the proof.

The problem is to encode a nonseparating curve in $\mathcal{C C}(S)$ in terms of homologically trivial curves. The main difficulty results from the "infiniteness" of this problem; for example, any nonseparating curve can be completed to a bounding pair in infinitely many different ways, none of which is more natural than any other. The key idea is to consider all possible completions at once.

Consider a vertex $\gamma$ of $\mathcal{T} \mathcal{G}(S)$ represented by a bounding pair of nonseparating curves $C_{0}, C_{1}$. We would like to single out one of these curves using only the 
information available in $\mathcal{T} \mathcal{G}(S)$. This can be done by using a separating curve $D$ which has $i\left(D, C_{0}\right) \neq 0$ and $i\left(D, C_{1}\right)=0$. The isotopy class $\left\langle C_{0}\right\rangle$ obviously can be recovered from the pair $(\gamma, \delta)$, where $\delta=\langle D\rangle$. Therefore the isotopy class $\left\langle C_{0}\right\rangle$ can be encoded by $(\gamma, \delta)$. For technical reasons, we use only pairs which we will call admissible (see below). Of course, we need to be able to tell when two such pairs encode the same isotopy class.

Definition (Admissible pair). Let $\gamma$ be a bounding pair and let $\delta$ be a separating curve. We call the pair $(\gamma, \delta)$ an admissible pair if both:

1. $\gamma$ is a vertex of some marked triangle $\left\{\gamma, \gamma^{\prime}, \beta\right\}$ of $T(S)$, and

2. $\delta$ is connected by an edge of $T(S)$ with $\beta$ and is not connected by an edge with either $\gamma$ or $\gamma^{\prime}$.

We will say that the triangle $\left\{\gamma, \gamma^{\prime}, \beta\right\}$ certifies the admissibility of the pair $(\gamma, \delta)$

Let the marked triangle $\left\{\gamma, \gamma^{\prime}, \beta\right\}$ be determined by three curves $C_{0}, C_{1}, C_{2}$ (as in the definition of the marked triangles), and suppose that $\beta$ is the isotopy class of the pair $C_{1}, C_{2}$ (and, hence, $\gamma, \gamma^{\prime}$ are the isotopy classes of two pairs including $C_{0}$ ). Then $\delta$ can be represented by a curve $D$ disjoint from both $C_{1}$ and $C_{2}$. Since $\delta$ is not connected by an edge with $\gamma$, we have $i\left(D, C_{0}\right) \neq 0$. It follows, in particular, that the isotopy class $\left\langle C_{0}\right\rangle$ of the nonseparating curve $C_{0}$ is uniquely determined by the pair $(\gamma, \delta)$. We will say that the isotopy class $\left\langle C_{0}\right\rangle$ is encoded by $(\gamma, \delta)$. Obviously, such an encoding of $\left\langle C_{0}\right\rangle$ is far from being unique. We will account for this non-uniqueness by using the following moves.

Two moves on admissible pairs: We consider the following two types of moves for admissible pairs $(\gamma, \delta)$.

Type I move: If a marked triangle $\left\{\gamma, \gamma^{\prime}, \beta\right\}$ certifies the admissibility of the pair $(\gamma, \delta)$, then replace $(\gamma, \delta)$ by $\left(\gamma^{\prime}, \delta\right)$.

Type II move: If a marked triangle $\left\{\gamma, \gamma^{\prime}, \beta\right\}$ certifies the admissibility of the pair $(\gamma, \delta)$ and simultaneously certifies the admissibility of the pair $\left(\gamma, \delta^{\prime}\right)$, then replace $(\gamma, \delta)$ by $\left(\gamma, \delta^{\prime}\right)$.

The key result about the encodings of nonseparating curves by homologically trivial ones is the following.

Theorem 5 (Equivalence of encodings). Two admissible pairs encode the same nonseparating curve if and only if they are connected by a sequence of moves of types I and II.

This theorem is deduced from the above connectedness theorems, namely from Theorems 3,4 .

Once this is proven, the proof that an automorphism of $\mathcal{T} \mathcal{G}(S)$ induces an automorphism of $\mathcal{C C}(S)$ follows easily, noting that the definition of admissible 
pairs and of moves of types I and II are given entirely in terms of the Torelli geometry.

Applications to the Torelli group. In order to state the main results about the Torelli groups, we need a counterpart of the extended mapping class group $\operatorname{Mod}^{ \pm}(S)$ for the symplectic groups. Namely, we need the extended symplectic group $\mathrm{Sp}^{ \pm}(2 g, \mathbf{Z})$, which is defined as the group of automorphisms of $\mathbf{Z}^{2 g}$ preserving the standard symplectic form up to an overall sign. Clearly, $\operatorname{Sp}^{ \pm}(2 g, \mathbf{Z})$ contains $\operatorname{Sp}(2 g, \mathbf{Z})$ as a subgroup of index 2 .

Let $S$ be a closed surface of genus $g$. The conjugation action of $\operatorname{Mod}^{ \pm}(S)$ on the normal subgroup $\mathcal{I}(S)$ induces homomorphisms

$$
\operatorname{Mod}^{ \pm}(S) \longrightarrow \operatorname{Aut}(\mathcal{I}(S)) \text { and } \operatorname{Sp}^{ \pm}(2 g, \mathbf{Z}) \longrightarrow \operatorname{Out}(\mathcal{I}(S))
$$

where $\operatorname{Aut}(\mathcal{I}(S))$ and $\operatorname{Out}(\mathcal{I}(S))$ are the groups of automorphisms and outer automorphisms, respectively, of $\mathcal{I}(S)$.

Our first application of Theorem 2 is the following.

Theorem 6 (Automorphisms of $\mathcal{I}(\boldsymbol{S})$ ). Let $S$ be a compact surface with $\operatorname{genus}(S) \geq 5$. Then the natural homomorphisms

$$
\operatorname{Mod}^{ \pm}(S) \longrightarrow \operatorname{Aut}(\mathcal{I}(S)) \quad \text { and } \quad \operatorname{Sp}^{ \pm}(2 g, \mathbf{Z}) \longrightarrow \operatorname{Out}(\mathcal{I}(S))
$$

are isomorphisms. In fact there is an isomorphism of exact sequences

$$
\begin{aligned}
& 1 \longrightarrow \mathcal{I}(S) \quad \longrightarrow \operatorname{Mod}^{ \pm}(S) \quad \longrightarrow \operatorname{Sp}^{ \pm}(2 g, \mathbf{Z}) \longrightarrow 1 \\
& \downarrow \approx \quad \downarrow \approx \quad \downarrow \approx \\
& 1 \longrightarrow \operatorname{Inn}(\mathcal{I}(S))) \longrightarrow \operatorname{Aut}(\mathcal{I}(S)) \longrightarrow \operatorname{Out}(\mathcal{I}(S)) \longrightarrow 1
\end{aligned}
$$

where the first exact sequence is an obvious version of the exact sequence (1) and the second exact sequence is the usual one for a centerless group, with Inn denoting the group of inner automorphisms induced by conjugation.

Note that the conclusion of Theorem 1 is false when $\operatorname{genus}(S)=2$, as Mess [Me] has shown that in this case $\mathcal{I}(S)$ is a countably generated free group. Theorem 6 was inspired by the theorem of Ivanov [Iv1], [Iv2] (see also [McC]) to the effect that $\operatorname{Out}\left(\operatorname{Mod}^{ \pm}(S)\right)=1$ for $\operatorname{genus}(S) \geq 3$.

The commensurator. Recall that the (abstract) commensurator group $\operatorname{Comm}(\Gamma)$ of a group $\Gamma$ is defined to be the set of equivalence classes of isomorphisms $\phi: H \rightarrow N$ between finite index subgroups $H, N$ of $\Gamma$, where $\phi_{1}: H_{1} \rightarrow N_{1}$ is equivalent to $\phi_{2}: H_{2} \rightarrow N_{2}$ if $\phi_{1}=\phi_{2}$ on some common finite index subgroup of both $H_{1}$ and $H_{2}$. The composition of homomorphisms induces a natural multiplication on $\operatorname{Comm}(\Gamma)$, which turns $\operatorname{Comm}(\Gamma)$ into a group.

The group $\operatorname{Comm}(\Gamma)$ is in general much larger than $\operatorname{Aut}(\Gamma)$; for example $\operatorname{Aut}\left(\mathbf{Z}^{n}\right)=\operatorname{GL}(n, \mathbf{Z})$ whereas $\operatorname{Comm}\left(\mathbf{Z}^{n}\right)=\operatorname{GL}(n, \mathbf{Q})$. The group $\operatorname{Comm}(\Gamma)$ 
was computed for mapping class groups of surfaces by Ivanov [Iv5]. For the Torelli group we have the following:

Theorem 7 (Commensurator Theorem). Let $S$ be a closed surface of genus $(S) \geq$ 5. Then the natural injection

$$
\operatorname{Mod}^{ \pm}(S) \longrightarrow \operatorname{Comm}\left(\mathcal{I}_{S}\right)
$$

is an isomorphism.

Non-arithmeticity. Our proof of the non-arithmeticity of $\mathcal{I}_{S}$ is inspired by the proof of the non-arithmeticity of $\operatorname{Mod}(S)$ given in [Iv5]. Theorem 7 implies that $\mathcal{I}_{S}$ is normal in its abstract commensurator. Using this fact, it is easy to see that $\mathcal{I}_{S}$ cannot be arithmetic. As an illustrating example, notice that the arithmetic group $\operatorname{GL}(n, \mathbf{Z})$ is not normal in its abstract commensurator $\operatorname{Comm}(\mathrm{GL}(n, \mathbf{Z}))=$ $\mathrm{GL}(n, \mathbf{Q})$.

The idea of proof of Theorems 6 and 7. The main step in the proof of Theorems 6 and 7 is to prove that the given isomorphism $\Phi: \Gamma_{1} \longrightarrow \Gamma_{2}$ between two subgroups of finite index in $\mathcal{I}_{S}$ induces an automorphism of the Torelli Geometry $\mathcal{T} \mathcal{G}(S)$; Theorem 2 can then be applied to produce a mapping class inducing $\Phi$. First of all, we note that the isotopy class of a curve is uniquely determined by (any non-zero power of) the Dehn twist about that curve. If one has a purely algebraic characterization of (the powers of) Dehn twists in the Torelli group, this can be used to produce an automorphism of $\mathcal{T} \mathcal{G}(S)$

We will denote the Dehn twist about a curve $\gamma$ by $T_{\gamma}$, and define the Dehn twist about a bounding pair $\{a, b\}$ as $T_{a b^{-1}}=T_{a} T_{b^{-1}}$. Dehn twist about a separating curve or bounding pair will be called a simple twist. For a group $\Gamma$ and element $f \in \Gamma$, let $C_{\Gamma}(f)$ denote the subgroup of elements $g \in \Gamma$ commuting with $f$, and let $Z(\Gamma)$ denote the center of $\Gamma$. The following proposition gives us an algebraic characterization of (powers of) simple twists.

Proposition 8 (Characterizing simple twists in $\mathcal{I}(S)$ ). Let $S$ be a closed surface with genus $(S) \geq 3$, and let $f \in \mathcal{I}(S)$ be nontrivial. Then $f$ is a power of a simple twist if and only if both of the following hold:

1. $Z\left(C_{\mathcal{I}(S)}(f)\right)=\mathbf{Z}$, and

2. The maximum of ranks of abelian subgroups of $\mathcal{I}(S)$ that contain $f$ is $2 g-3$.

From Proposition 8 one can easily deduce that any isomorphism $\Phi: \Gamma_{1} \longrightarrow$ $\Gamma_{2}$ between two subgroups $\Gamma_{1}, \Gamma_{2}$ of finite index in $\mathcal{I}_{S}$ takes powers of simple twists to powers of simple twists. The main tool in proving Proposition 8 is the Thurston normal form theory for mapping classes extended to abelian subgroups of $\operatorname{Mod}(S)$, for example, in [Iv4].

Next, we need further to distinguish purely algebraically between the two types of simple twists.

Proposition 9 (Characterizing bounding pairs). Let $g \geq 4$, and let $R$ be the set of powers of simple twists in $\mathcal{I}(S)$. Then the following are equivalent. 
1. $f \in R$ is a power of a Dehn twist about a bounding pair.

2. There exist $g, h \in R$, distinct from $f$ and from each other, so that the group generated by $f, g, h$ is isomorphic to $\mathbf{Z}^{2}$.

Finally, we need to give a purely algebraic characterization of marked triangles in $\mathcal{T} \mathcal{G}(S)$.

Proposition 10 (Characterizing marked triangles). Let $g \geq 4$, and let $R$ be the set of powers of simple twists in $\mathcal{I}(S)$. Then the following are equivalent.

1. $f, g, h \in R$ are powers of a Dehn twists about a bounding pairs $\gamma_{1}, \gamma_{2}, \gamma_{3}$ such that $\left\{\gamma_{1}, \gamma_{2}, \gamma_{3}\right\}$ is a marked triangle.

2. The groups generated by elements $f, g, h$ and by any two of them are all isomorphic to $\mathbf{Z}^{2}$.

The proofs of the last two Propositions are similar, and are based on an analysis of all possible configurations of three separating circles and bounding pairs on a surface.

\section{References}

[BLM] J. Birman, A. Lubotzky, and J. McCarthy, Abelian and solvable subgroups of the mapping class group, Duke Math. Jour., 50 (1983), 1107-1120.

$[\mathrm{BM}] \quad$ T. Brendle and D. Margalit, Commensurations of the Johnson kernel, Geometry and Topology, 8 (2004), 1361-1384.

[FI] B. Farb and N. V. Ivanov, Torelli geometry and commensurations of the Torelli group, in preparation.

[FM] B. Farb and H. Masur, Superrigidity and mapping class groups, Topology, 37 (1998), 1169-1176.

[Hain] R. M. Hain, Torelli groups and geometry of moduli spaces of curves, In: Current topics in algebraic geometry (Berkeley, CA, 1992/93), MSRI Publications, 28, Cambridge University Press, 1995, 97-143.

[Harv] W. J. Harvey, Boundary structure of the modular group, In: Riemann surfaces and related topics: Proceedings of the 1978 Stony Brook Conference, Ed. by I. Kra and B. Maskit, Annals of Math. Studies, 97, Princeton University Press, 1981, 245-251.

[Iv1] N. V. Ivanov, Algebraic properties of the Teichmüller modular group, DAN SSSR, 275 (1984), 786-789; English transl.: Soviet Mathematics-Doklady, 29 (1984), $288-291$.

[Iv2] _ Automorphisms of Teichmüller modular groups, Lecture Notes in Math., 1346 (1988), 199-270.

[Iv3] Teichmüller modular groups and arithmetic groups, In: Research in Topology. 6, Notes of LOMI scientific seminars, 167 (1988), 95-110; English transl.: J. Soviet Math., 52 (1990), 2809-2818.

[Iv4] _ Subgroups of Teichmüller Modular Groups, Translations of Mathematical Monographs, 115, AMS, 1992.

[Iv5] _ Automorphisms of complexes of curves and of Teichmüller spaces, International Mathematics Research Notices, bf 14 (1997), 651-666.

[IM] N. V. Ivanov and J. D. McCarthy, On injective homomorphisms between Teichmüller modular groups. I, Invent. Math. 135 (1999), 425-486.

[Jo1] D. Johnson, A survey of the Torelli group, Contemp. Math., 20 (1983), 165-179.

[Jo2] D. Johnson, The structure of the Torelli group I: A finite set of generators for $\mathcal{T}$, Annals of Math. 118 (1983), 423-442. 
[McC] J. D. McCarthy, Automorphisms of surface mapping class groups. A recent theorem of $N$. Ivanov, Invent. Math., 84 (1986), 49-71.

$[\mathrm{McV}] \quad$ J. D. McCarthy and W. Vautaw, Automorphisms of Torelli groups, preprint, Nov. 2003.

[Me] G. Mess, The Torelli group for genus 2 and 3 surfaces, Topology 31 (1992), 775790 .

[MS] H. Masur and S. Schleimer, The pants complex has only one end, preprint, 2004.

Dept.of Mathematics, University of Chicago, 5734 University Ave., Chicago, IL 60637

E-mail address: farb@math.uchicago.edu

Dept. Of Math., Wells Hall, Michigan State University, East Lansing, MI 488241027

E-mail address: ivanov@math.msu.edu 